\title{
Energy Efficient Design for Two-Way AF Relay Networks
}

\author{
Yong Li, ${ }^{1}$ Zhengguang Zheng, ${ }^{2}$ Mingyue Zhao, ${ }^{3}$ Yunli Chen, ${ }^{1}$ and Chuanyong Liu ${ }^{2}$ \\ ${ }^{1}$ Institute of Embedded Software and Systems, Beijing University of Technology, Beijing 100022, China \\ ${ }^{2}$ Department of Vehicle Information Systems, North Information Control Group Co., Ltd., Nanjing 211153, China \\ ${ }^{3}$ Department of Physical, No. 1 High School of Shunde, Foshan 528300, China
}

Correspondence should be addressed to Zhengguang Zheng; zhguangzheng@gmail.com

Received 21 August 2013; Revised 9 December 2013; Accepted 26 December 2013; Published 28 January 2014

Academic Editor: Athanasios Panagopoulos

Copyright (C) 2014 Yong Li et al. This is an open access article distributed under the Creative Commons Attribution License, which permits unrestricted use, distribution, and reproduction in any medium, provided the original work is properly cited.

\begin{abstract}
Conventional designs on two-way relay networks mainly focus on the spectral efficiency (SE) rather than energy efficiency (EE). In this paper, we consider a system where two source nodes communicate with each other via an amplify-and-forward (AF) relay node and study the power allocation schemes to maximize EE while ensuring a certain data rate. We propose an optimal energyefficient power allocation algorithm based on iterative search technique. In addition, a closed-form suboptimal solution is derived with reduced complexity and negligible performance degradation. Numerical results show that the proposed schemes can achieve considerable EE improvement compared with conventional designs.
\end{abstract}

\section{Introduction}

The unprecedented growth of wireless networks has resulted in a rapid rise of energy consumption and led to an emerging trend of green radio [1]. Energy-efficient system design for green radio is earning considerable attention from both industry and academia for its positive impact on environment. The energy efficiency (EE) [2], which is widely defined as the system throughput per unit energy, has become one of the critical performance metrics for future communication systems.

In the green communication systems, several advanced wireless communication techniques, such as cooperative relay transmissions $[3,4]$ and small cells $[5,6]$, have been adopted to provide significant capacity improvements and reduce energy consumption. Compared with direct transmission, relay technique is essential to provide a more reliable transmission due to the smaller path loss attenuation of shorter hops. The two-way relay system was introduced in [7] for two source nodes which want to exchange information with each other via a relay.

Most previous works on two-way relay networks mainly focused on the relay selection or power allocation from the perspective of spectral efficiency (SE) $[8,9]$. In [8], the smaller of the received signal-to-noise ratios (SNRs) of two transceivers was maximized through joint relay selection and power allocation. Achievable data rate region for the two source nodes was obtained in [9], and considerable SE enhancement was achieved when compared with one-way relay network. However, the EE is a strictly decrease function of SE when only transmit power is considered [1]. After taking the circuit power used for electronic devices and signal processing into account, the relation between SE and EE is more complicated. This indicates that maximizing SE may not maximize $\mathrm{EE}$ in a practical two-way relay system.

Recently, some works have investigated energy related designs in two-way relay networks [10-15]. An efficient power allocation scheme to minimize the total transmit power consumption was presented in [10] for the machine-tomachine networks, where the source node communicated with its corresponding destination node via one or more relays. The authors in [12] proposed a transmission scheme to minimize the energy consumed per bit transmitted for both one-way and two-way relay networks by joint relay selection and power allocation. Reference [13] investigated a joint relay selection and power allocation scheme to minimize overall transmit power while ensuring a certain data rate. However, the circuit power was ignored in all of them. In a practical system, not only the transmit power but also the circuit power contributes to the total power consumption. 
Therefore, minimizing the transmit power may not necessarily lead to a high EE [14]. In [15], after taking the realistic power consumption model into consideration, an analytical framework for the total energy consumption of AF multihop network while satisfying an average bit error rate requirement was developed. Based on this framework, the impact of the relay's location and energy resource allocation between the relay and the source were also evaluated. The EE of two-way and one-way relay systems was analyzed in [11] and the search technique was used to find the optimal solution which had high complexity and incurred additional energy.

In this paper, we propose an optimal energy-efficient power allocation algorithm for two-way relay networks while ensuring a certain throughput requirement. Using the results derived in [14], we first study the transmit power minimization problem for a fixed data rate, analyze the relation between SE and EE, and then apply this solution to formulate an equivalent optimization problem with one variable. To solve this problem, bisection search technique is used. However, the optimal power allocation with iterative search method has high computational complexity. In order to reduce the complexity, a closed-form solution of suboptimal power allocation algorithm is derived. The proposed algorithm not only eliminates the complexity of bisection search, but also can achieve near optimal EE capacity performance in two-way relay networks.

The rest of the paper is organized as follows. Section 2 introduces a two-way relay network model and formulates the corresponding power allocation problem. In Section 3, both the optimal and suboptimal power allocation schemes are proposed. Simulation results are given in Section 4. Section 5 concludes this paper.

\section{System Model and Problem Formulation}

2.1. System Model. Consider a relay network consisting of two source nodes $\mathbb{A}$ and $\mathbb{B}$ exchanging information with each other at the same transmission rate via a half-duplex AF relay $\mathbb{R}$. All the nodes are equipped with a single antenna. Assume that the signal is severely attenuated between two source nodes because of the high shadowing caused by obstacles or long distance, and thus the direct link transmission is not considered here.

The channels among three nodes are assumed as block fading channels and the perfect channel state information (CSI) is available at each node. Denote by $h_{a r}$ and $h_{b r}$ the channel coefficients from source nodes $\mathbb{A}$ and $\mathbb{B}$ to relay node $\mathbb{R}$, respectively. To exchange information from both of two source nodes, $\mathbb{A}$ and $\mathbb{B}$ must transmit in two phases. In the multiple access phase, both of the two source nodes $\mathbb{A}$ and $\mathbb{B}$ simultaneously transmit their respective unit-energy symbols $s_{a}$ and $s_{b}$ to the relay node $\mathbb{R}$. Let $P_{a}$ and $P_{b}$ be the transmit power of source nodes $\mathbb{A}$ and $\mathbb{B}$, respectively. Thus the received signal can be expressed as

$$
r=\sqrt{P_{a}} h_{a r} s_{a}+\sqrt{P_{b}} h_{b r} s_{b}+z_{r}
$$

where $z_{r}$ is the noise at relay node $\mathbb{R}$. All the noise terms in this paper are assumed to be independent Gaussian variables with zero mean and a variance of $\sigma^{2}$.

In the broadcast phase, $\mathbb{R}$ transmits the received signal $r$ to $\mathbb{A}$ and $\mathbb{B}$ with an amplification factor $\xi$. The received signals $y_{a}$ and $y_{b}$ at both of the two source nodes can be written as

$$
\begin{gathered}
y_{a}=\xi h_{a r} r+z_{a}, \\
y_{b}=\xi h_{b r} r+z_{b}, \\
\xi=\sqrt{\frac{P_{r}}{P_{a}\left|h_{a r}\right|^{2}+P_{b}\left|h_{b r}\right|^{2}+\sigma^{2}}},
\end{gathered}
$$

where $z_{i}$ is the additive noise at the source node $i(i \in\{a, b\})$ and $P_{r}$ is the transmit power of relay node $\mathbb{R}$. Since each source node receives a copy of its own transmitted signal as interference, the partner's message can be decoded after selfinterference cancelation (SIC).

2.2. Problem Formulation. Define parameters $g_{a r}=\left|h_{a r}\right|^{2} / \sigma^{2}$ and $g_{b r}=\left|h_{b r}\right|^{2} / \sigma^{2}$ as the instantaneous channel gain-tonoise ratio (CNR); then the received SNR at two source nodes can be given as

$$
\begin{aligned}
& \gamma_{a b}=\frac{g_{a r} g_{b r} P_{a} P_{r}}{g_{a r} P_{a}+g_{b r}\left(P_{b}+P_{r}\right)+1}, \\
& \gamma_{b a}=\frac{g_{a r} g_{b r} P_{b} P_{r}}{g_{b r} P_{b}+g_{a r}\left(P_{a}+P_{r}\right)+1} .
\end{aligned}
$$

The instantaneous transmission data rate of nodes $\mathbb{A}$ to $\mathbb{B}$ can be obtained from the Shannon capacity formulae, which are, respectively,

$$
\begin{aligned}
& R_{a b}=\frac{1}{2} B \log _{2}\left(1+\gamma_{a b}\right), \\
& R_{b a}=\frac{1}{2} B \log _{2}\left(1+\gamma_{b a}\right),
\end{aligned}
$$

where $B$ is the transmission bandwidth. Hence, the overall data rate can be driven as

$$
R=R_{a b}+R_{b a} .
$$

According to [16], the circuit power consumption $P_{c}$ is incurred by signal processing and active electronic devices in the network, and it can be modeled as a linear function of transmission rate

$$
P_{c}=P_{s}+\beta R
$$

where $P_{s}$ is the static circuit power and $\beta$ is the dynamic circuit power per unit data rate. Thus the total power consumption $P_{t}$ is

$$
P_{t}=\frac{\alpha\left(P_{A}+P_{B}+P_{R}\right)}{2}+P_{c},
$$


where $\alpha$ is a constant related to the efficiency of power amplifier.

In order to guarantee the quality of service (QoS), the transmission rate should be restricted by

$$
R \geq \check{R}
$$

where $\check{R}$ is the minimum data rate requirement related to the type of traffic.

Since the total transmit power $P_{i}$ at node $i$ is limited, we have

$$
P_{i} \leq \widehat{P}_{i}, \quad i \in\{a, b, r\},
$$

where $\widehat{P}_{i}$ is the maximum allowable transmit power.

The objective of this paper is to find the optimal power allocation of each node to maximize the EE of two-way relay network while ensuring a certain data rate. Hence, the optimization problem can be formulated as follows $(P 1)$ :

$$
\begin{array}{rl}
P 1: \max _{P_{i}} & U=\frac{R}{P_{t}} \\
\text { s.t. } & R_{a b}=R_{b a}, \\
& R \geq \check{R}, \\
& P_{i} \leq \widehat{P}_{i}, \quad i \in\{a, b, r\} .
\end{array}
$$

We assume that $\check{R}$ can be achieved under constraint (10d). If not, the feasible solution of problem $P 1$ does not exist. In this case, the scheduler may have to decrease $\breve{R}$ to make the solution feasible.

\section{Energy-Efficient Design}

In this section, we aim to find the optimal transmit powers $P_{a}^{*}, P_{b}^{*}$, and $P_{r}^{*}$ to maximize EE while satisfying a certain data rate. Before solving the problems (10a), (10b), (10c), and (10d), we introduce two auxiliary optimization problems below. Define the transmit power minimization $(\mathrm{MinP})$ problem $\mathrm{P} 2$ under the required minimum data rate constraint as

$$
\begin{array}{cl}
P 2: \min _{P_{i}} & P_{a}+P_{b}+P_{r} \\
\text { s.t. } & R_{a b}=R_{b a}, \\
& R \geq \breve{R} .
\end{array}
$$

Using the similar approach in [14], we have the optimal solution to problem $P 2$ :

$$
\begin{aligned}
& P_{a}=f_{a}(\check{\phi})=\frac{\check{\phi}}{g_{a r}}+\check{\phi} \sqrt{\frac{2 \check{\phi}+1}{2 \check{\phi} g_{a r} g_{b r}}}, \\
& P_{b}=f_{b}(\check{\phi})=\frac{\check{\phi}}{g_{b r}}+\check{\phi} \sqrt{\frac{2 \check{\phi}+1}{2 \check{\phi} g_{a r} g_{b r}}},
\end{aligned}
$$

$$
P_{r}=f_{r}(\check{\phi})=\check{\phi}\left(\frac{1}{g_{a r}}+\frac{1}{g_{b r}}\right)+2 \check{\phi} \sqrt{\frac{2 \check{\phi}+1}{2 \check{\phi} g_{a r} g_{b r}},}
$$

where $\check{\phi}=2^{\check{R} / B}-1$.

The other conventional optimization problem is data rate maximization $(\operatorname{Max} R)$ problem $P 3$ subject to the total transmit power constraint. It can be described as follows

$$
\begin{array}{rl}
P 3: \max _{P_{i}} & R \\
\text { s.t. } & R_{a b}=R_{b a}, \\
& P_{i} \leq \widehat{P}_{i}, \quad i \in\{a, b, r\} .
\end{array}
$$

According to (12a), (12b), and (12c), it can be seen that both the overall data rate $R$ and transmit power $P_{i}$ at node $i$ are the strictly increasing function of $\phi$. Thus we can obtain the maximum achievable data rate $\widehat{R}$

$$
\widehat{R}=B \log _{2}(1+\widehat{\phi}),
$$

where $\widehat{\phi}=\min _{i \in\{a, b, r\}} f_{i}^{-1}\left(\widehat{P}_{i}\right)$ and $f_{i}^{-1}(\cdot)$ is the inverse function of $f_{i}(\cdot)$.

Since we assume that $\breve{R}$ is achievable under the constraints (10b) and (10d), we have $\check{\phi} \leq \widehat{\phi}$. The equivalent optimization problem of $P 1$ can be reformulated as

$$
P 4: \max _{\phi} U(\phi)=\frac{R(\phi)}{\alpha \sum_{i \in\{a, b, r\}}\left(f_{i}(\phi) / 2\right)+P_{s}+\beta R(\phi)}
$$

$$
\text { s.t. } \quad \breve{\phi} \leq \phi \leq \widehat{\phi},
$$

where $R(\phi)=B \log _{2}(1+\phi)$.

According to [16], we can prove that the system $\operatorname{EE} U(\phi)$ is a quasiconcave function of $\phi(\phi>0)$.

3.1. Optimal Energy-Efficient Power Allocation. Since the EE $U$ is a quasiconcave function of $\phi$, an optimal solution $\widetilde{\phi}$ that maximizes $\mathrm{EE}$ without any constraint always exists. Then $U(\phi)$ is an increasing function of $\phi$ when $0 \leq \phi \leq \widetilde{\phi}$ and $U(\phi)$ is decreasing when $\phi \geq \widetilde{\phi}$. The optimal solution $\phi^{*}$ for problem $P 4$ is given as

$$
\phi^{*}= \begin{cases}\widehat{\phi}, & \check{\phi} \leq \widehat{\phi} \leq \widetilde{\phi} \\ \widetilde{\phi}, & \check{\phi} \leq \widetilde{\phi} \leq \widehat{\phi} \\ \check{\phi}, & \widetilde{\phi} \leq \check{\phi} \leq \widehat{\phi} .\end{cases}
$$

It is noticeable that (16) is equivalent to

$$
\phi^{*}=\min (\widehat{\phi}, \max (\widetilde{\phi}, \check{\phi})) .
$$

The remaining thing for problem $P 4$ is to address $\phi^{*}$ and it can be solved by bisection search technique. Here we 
Step 1. Initialization, $\phi_{m}=0, \phi_{l}=0, \phi_{h}=0$.

Step 2. Calculate $\check{\phi}$ and $\widehat{\phi}$ according to (12a)-(12c) and (14). Set $\phi_{l}=\check{\phi}$ and $\phi_{h}=\widehat{\phi}$.

Step 3. Set $\phi_{m}=\left(\phi_{l}+\phi_{h}\right) / 2$.

Step 4. If its first derivative with respect to $\phi$ satisfies $\left.(d U(\phi) / d \phi)\right|_{\phi=\phi_{m}}>0$, then $\phi_{l}=\phi_{m}$, else $\phi_{h}=\phi_{m}$.

Step 5. Repeat Step 3 until $\phi_{h}-\phi_{l} \leq \varepsilon$ where $\varepsilon$ is a small positive constant to control the convergence accuracy.

Step 6. Calculate the optimal solution $P_{i}^{*}=f_{i}\left(\phi_{m}\right), i=\{a, b, r\}$ according to (12a)-(12c).

Algorithm 1: Optimal power allocation (OPA) algorithm.

list the optimal power allocation method in Algorithm 1. In each iteration, the search region is divided into two parts. To update the search region, we should determine which part contains $\tilde{\phi}$ by calculating the first derivative of $U(\phi)$. Note that the sign of $\mathrm{d} U(\phi) / \mathrm{d} \phi$ can be determined by calculating the value of $U(\phi+\Delta \phi)-U(\phi)$, where $\Delta \phi$ is an infinitely small positive constant.

3.2. Suboptimal Energy-Efficient Power Allocation. Although the optimal power allocation solution is desirable, the cost to pay is the computational complexity in iterative search. It takes at most $\left\lceil\log _{2}((\widehat{\phi}-\check{\phi}) / \varepsilon)\right\rceil$ iterations to convergence which incurs additional energy consumption, where $\lceil x\rceil$ denotes the smallest integer not less than $x$. Thus, this straightforward approach is clearly not practical, and we need a low complexity approach to solve this problem.

Considering the following approximation for large $\phi$ :

$$
\sqrt{2 \phi(2 \phi+1)} \approx 2 \phi+\frac{1}{2}
$$

then the EE of problem $P 4$ can be written as

$$
\begin{aligned}
U(\phi) & =\frac{R(\phi)}{\alpha \sum_{i \in\{a, b, r\}}\left(f_{i}(\phi) / 2\right)+P_{s}+\beta R(\phi)} \\
& \approx \frac{B \log _{2}(1+\phi)}{\phi G_{1}+G_{2}+\beta B \log _{2}(1+\phi)},
\end{aligned}
$$

where $G_{1}=\alpha\left(\left(1 / \sqrt{g_{a r}}\right)+\left(1 / \sqrt{g_{b r}}\right)\right)^{2}$ and $G_{2}=(\alpha / 2$ $\left.\sqrt{g_{a r} g_{b r}}\right)+P_{s}$. It can be easily proved that $\widetilde{U}(\phi)$ is also a quasiconcave function of $\phi$; the unique solution $\widetilde{\phi}$ for achieving the maximum $U(\phi)$ without any constraint always exists. Differentiating (19) with respect to $\phi$ and setting this derivative to zero, we can obtain

$$
\frac{\mathrm{d} \widetilde{U}}{\mathrm{~d} \phi}=F\left(\frac{\phi+\left(G_{2} / G_{1}\right)}{1+\phi}-\ln (1+\phi)\right)=0,
$$

where

$$
F=\frac{B}{G_{1}\left(\phi G_{1}+G_{2}+\beta B \log _{2}(1+\phi)\right)^{2} \ln 2} .
$$

Since $F$ is a positive variable, equation (20) can be written as

$$
\phi+\frac{G_{2}}{G_{1}}=(1+\phi) \ln (1+\phi)
$$

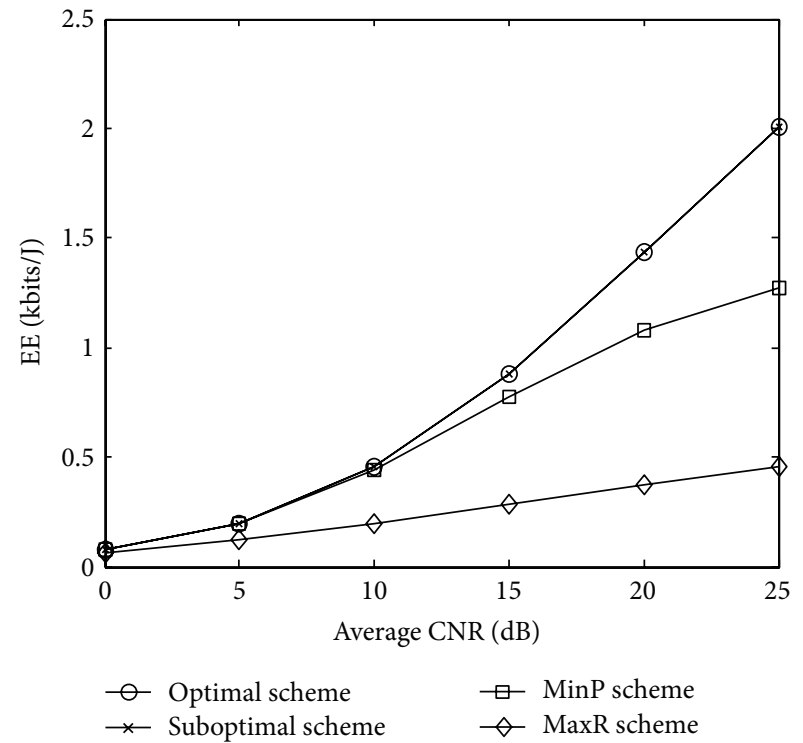

Figure 1: System EE versus average CNR.

After some mathematical manipulation, we can find the root $\widetilde{\phi}$ of (22), which is given as

$$
\tilde{\phi}=\exp \left(W_{0}\left(\frac{G_{2}}{G_{1} e}-\frac{1}{e}\right)+1\right)-1,
$$

where $e \approx 2.7183$ is the base of the natural logarithm and $W_{0}(\cdot)$ denotes the real branch of the Lambert function [17]. Thus the suboptimal power allocation can be obtained by substituting $\widetilde{\phi}$ into (17), (12a), (12b), and (12c). The suboptimal transmit power $P_{i}^{*}$ at node $i$ is

$$
P_{i}^{*}=f_{i}\left(\phi^{*}\right), \quad i \in\{a, b, r\} .
$$

\section{Numerical Results}

In this section, we provide simulations to evaluate the EEs of our proposed schemes and validate the previous analysis. The channel gains $h_{a r}$ and $h_{b r}$ are assumed to be independent block Rayleigh fading with the same average CNR. Simulation parameters are set as follows: $B=1 \mathrm{kHz}, \widehat{P}_{a}=\widehat{P}_{b}=5 \mathrm{~W}$, $\widehat{P}_{r}=10 \mathrm{~W}, P_{s}=0.5 \mathrm{~W}, \alpha=2, \check{R}=1 \mathrm{kbps}$, and $\beta=$ $0.2 \mathrm{~W} / \mathrm{kbps}$.

The system EE and corresponding data rate versus average CNR are evaluated in Figures 1 and 2 under different 


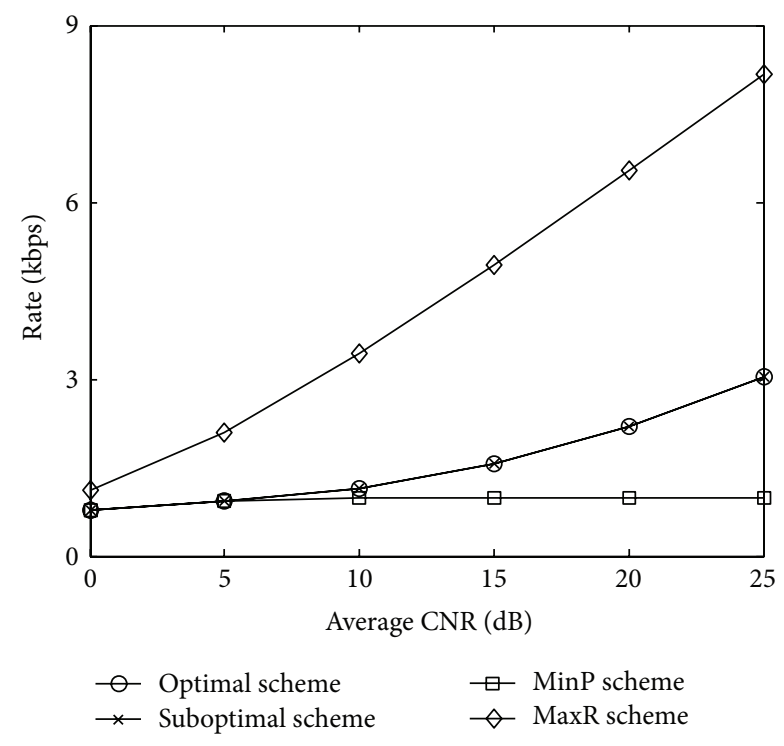

Figure 2: System rate versus average CNR.

strategies. It can be seen that both $\mathrm{EE}$ and data rate increase with the average CNR. This is because the energy-efficient design tends to use less transmit power when CNR increases. Figure 1 shows that the proposed schemes outperform conventional schemes and the suboptimal scheme achieves near optimal performance. The performance gap between proposed schemes and conventional schemes becomes larger when CNR increases. In low CNR region, the transmit power dominates the total power consumption while in the high CNR region the circuit power does for the energy-efficient design. From Figure 2, it indicates that for specific channel realizations with bad quality, energy-efficient design may have to operate exactly at the maximum transmit power to meet the minimum rate requirement. It can be seen that the proposed schemes have lower SE than the MaxR scheme and can achieve higher EE with less power consumption at the cost of some SE loss when CNR is large enough. This indicates that our proposed schemes can achieve a better tradeoff between EE and SE.

\section{Conclusion}

In this paper, we studied the energy-efficient design of twoway networks under the constraints of transmit power and the minimum rate requirement. We proposed an optimal power allocation scheme based on iterative search method. In order to reduce the computational complexity, we also derived a closed-form solution for suboptimal algorithm that performs near the optimal scheme. Compared with traditional SE-oriented power allocation, the proposed schemes have significant improvement in terms of EE.

\section{Conflict of Interests}

The authors declare that there is no conflict of interests regarding the publication of this paper.

\section{Acknowledgment}

This work is partially supported by the funding from Beijing Natural Science Foundation (no. 4122009).

\section{References}

[1] Y. Chen, S. Zhang, S. Xu, and G. Y. Li, "Fundamental trade-offs on green wireless networks," IEEE Communications Magazine, vol. 49 , no. 6 , pp. 30-37, 2011.

[2] D. Feng, C. Jiang, G. Lim, L. J. Cimini, Jr., G. Feng, and G. Y. Li, "A survey of energy-efficient wireless communications," IEEE Communications Surveys and Tutorials, vol. 15, no. 1, pp. 167178, 2013.

[3] V. K. Sakarellos, C. I. Kourogiorgas, D. Skraparlis, A. D. Panagopoulos, and J. D. Kanellopoulos, "End-to-end performance analysis of millimeter wave triple-hop backhaul transmission systems," Wireless Personal Communications, vol. 71, no. 4, pp. 2725-2740, 2013.

[4] V. K. Sakarellos, D. Skraparlis, A. D. Panagopoulos, and J. D. Kanellopoulos, "Cooperative diversity performance in millimeter wave radio systems," IEEE Transactions on Communications, vol. 60, no. 12, pp. 3641-3649, 2012.

[5] H. Wang, J. Jiang, J. Li, M. Ahmed, and M. Peng, "High energy efficient heterogeneous networks: cooperative and cognitive techniques," International Journal of Antennas and Propagation, vol. 2013, Article ID 231794, 7 pages, 2013.

[6] V. K. Sakarellos, D. Skraparlis, and A. D. Panagopoulos, “Cooperation within the Small Cell: the indoor, correlated shadowing case," Physical Communication, vol. 9, pp. 16-22, 2013.

[7] P. Larsson, N. Johansson, and K.-E. Sunell, "Coded bidirectional relaying," in Proceedings of the IEEE 63rd Vehicular Technology Conference (VTC '06), pp. 851-855, May 2006.

[8] S. Talwar, Y. Jing, and S. Shahbazpanahi, "Joint relay selection and power allocation for two-way relay networks," IEEE Signal Processing Letters, vol. 18, no. 2, pp. 91-94, 2011.

[9] P. Popovski and H. Yomo, "Physical network coding in two-way wireless relay channels," in Proceedings of the IEEE International Conference on Communications (ICC'07), pp. 707-712, Glasgow, Scotland, June 2007.

[10] G. A. Elkheir, A. S. Lioumpas, and A. Alexiou, "Energy efficient AF relaying under error performance constraints with application to M2M networks," in Proceedings of the IEEE 22nd International Symposium on Personal, Indoor and Mobile Radio Communications ( PIMRC '11), pp. 56-60, September 2011.

[11] C. Sun and C. Yang, "Energy efficiency analysis of one-way and twoway relay systems," EURASIP Journal on Wireless Communications and Networking, vol. 2012, article 46, 2012.

[12] R. Huang, C. Feng, T. Zhang, and W. Wang, "Energy-efficient relay selection and power allocation scheme in AF relay networks with bidirectional asymmetric traffic," in Proceedings of the 14th International Symposium on Wireless Personal Multimedia Communications: Communications, Networking and Applications for the Internet of Things (WPMC '11), pp. 7-11, October 2011.

[13] M. Xu, Y. Wang, G. Li, and W. Lin, "On the energy-efficient power allocation for amplify-and-forward two-way relay networks," in Proceedings of the IEEE 74th Vehicular Technology Conference (VTC Fall '11), pp. 1-5, September 2011.

[14] C. Sun and C. Yang, "Is two-way relay more energy efficient?" in Proceedings of the 54th Annual IEEE Global Telecommunications 
Conference: "Energizing Global Communications" (GLOBECOM '11), pp. 1-6, December 2011.

[15] O. Waqar, M. A. Imran, M. Dianati, and R. Tafazolli, "Energy consumption analysis and optimization of BER-constrained amplify-and-forward relay networks," IEEE Transactions on Vehicular Technology, 2013.

[16] C. Xiong, G. Y. Li, S. Zhang, Y. Chen, and S. Xu, "Energy- and spectral-efficiency tradeoff in downlink OFDMA networks," IEEE Transactions on Wireless Communications, vol. 10, no. 11, pp. 3874-3886, 2011.

[17] R. M. Corless, G. H. Gonnet, D. E. G. Hare, D. J. Jeffrey, and D. E. Knuth, "On the Lambert W function," Advances in Computational Mathematics, vol. 5, no. 4, pp. 329-359, 1996. 

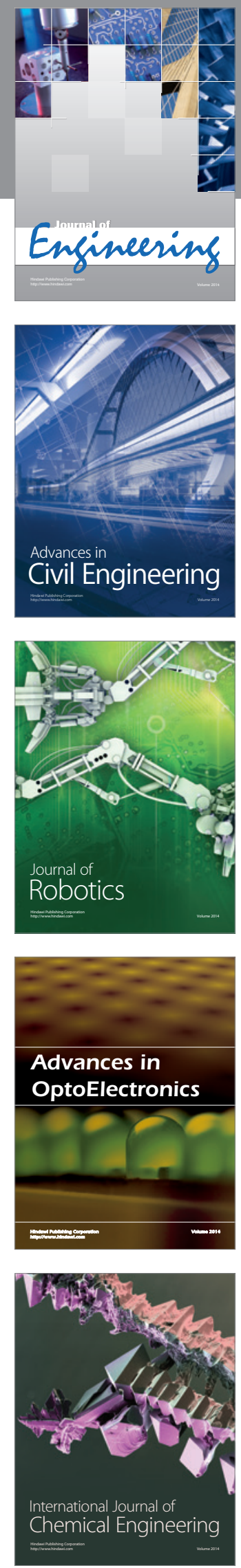

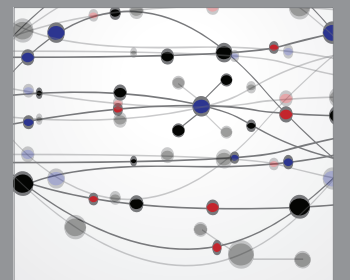

The Scientific World Journal
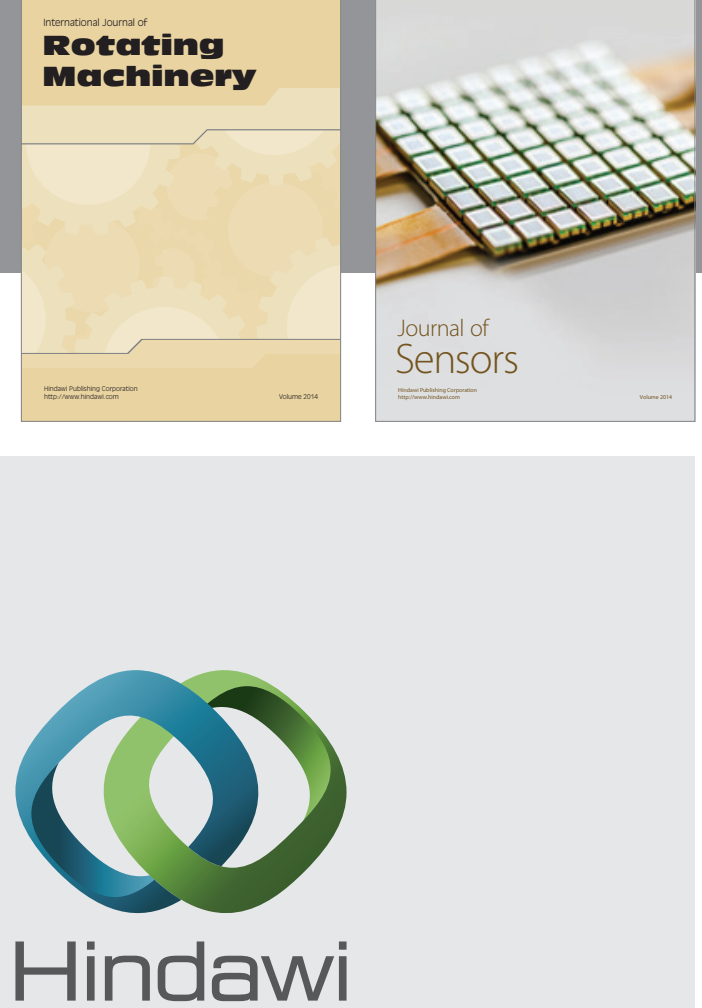

Submit your manuscripts at http://www.hindawi.com
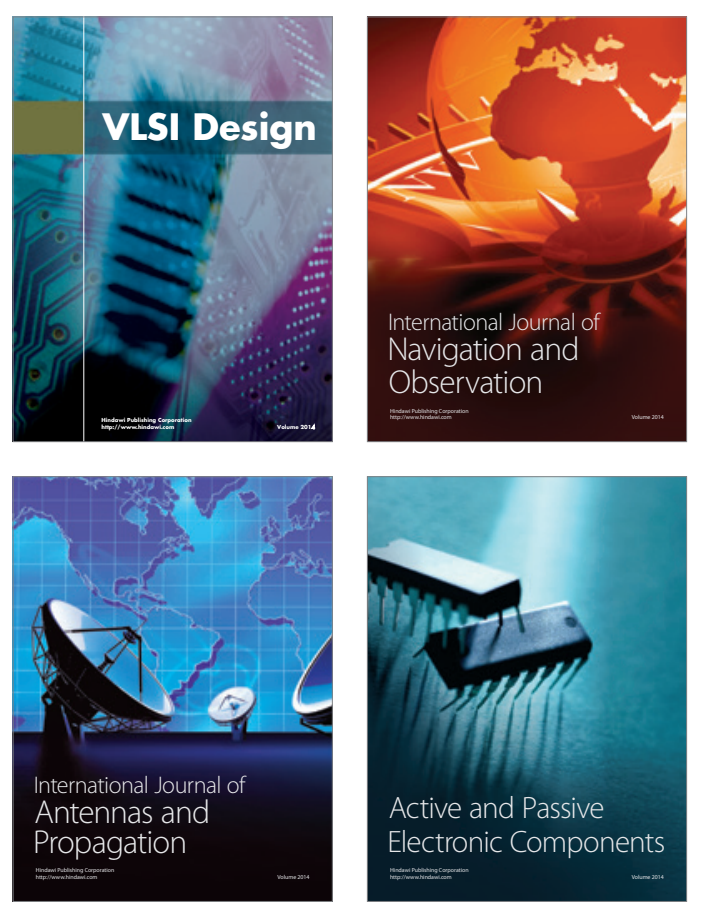
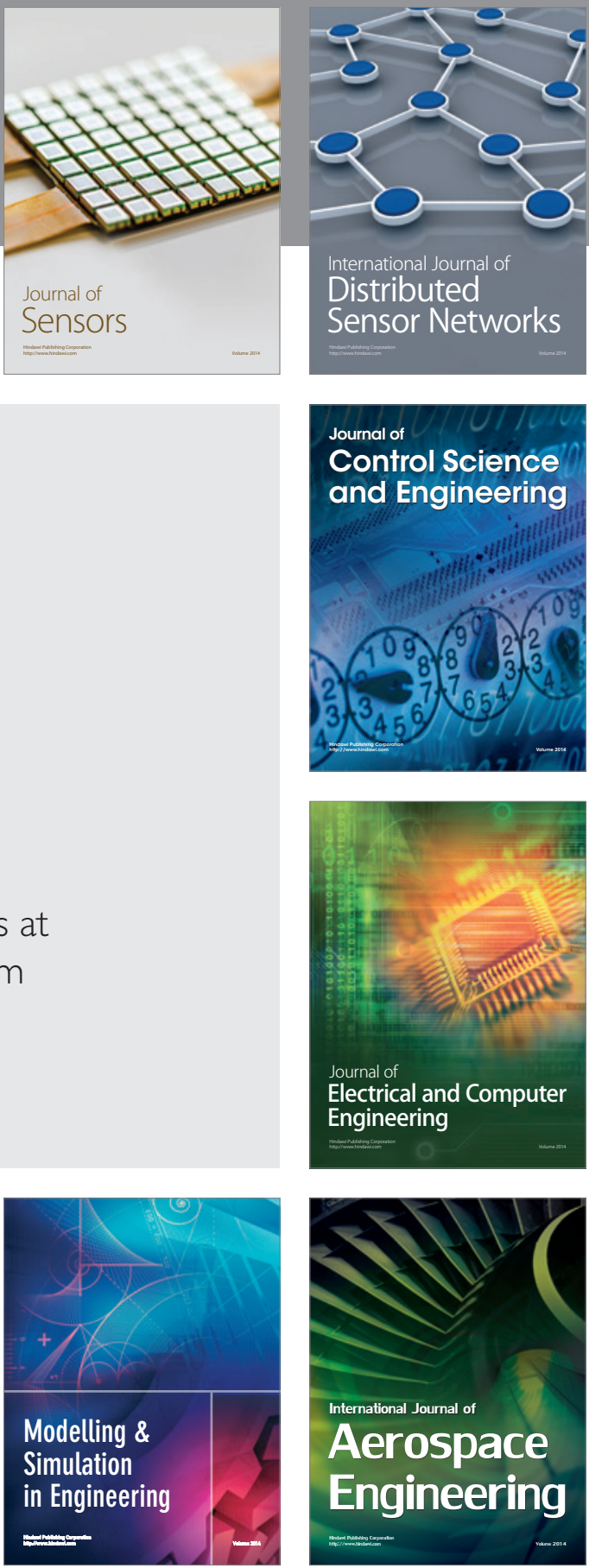

Journal of

Control Science

and Engineering
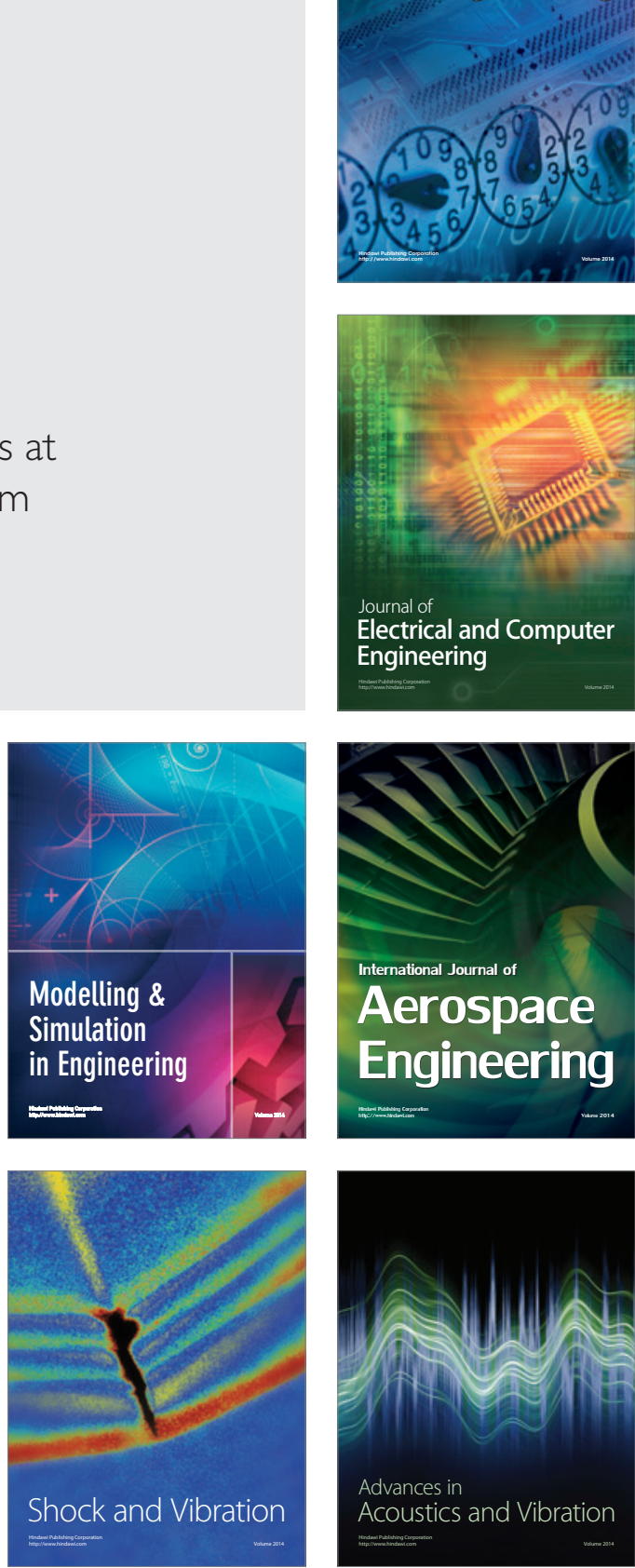